\title{
A possible origin of dayside Pc1 magnetic pulsations observed at high latitudes
}

\author{
V. Safargaleev ${ }^{1,2}$, A. Serebryanskaya ${ }^{1}$, A. Koustov ${ }^{3}$, M. Lester ${ }^{4}$, E. Pchelkina ${ }^{1}$, and A. Vasilyev ${ }^{1}$ \\ ${ }^{1}$ Polar Geophysical Institute, Fersman Str. 14, Apatity, 184200, Russia \\ ${ }^{2}$ Swedish Institute of Space Physics, Box 812, S-981 28, Kiruna, Sweden \\ ${ }^{3}$ University of Saskatchewan, 116 Science Place, Saskatoon, SK S7N 5E2, Canada \\ ${ }^{4}$ University of Leicester, University Road Leicester LE1 7RH, UK
}

Received: 18 August 2003 - Revised: 6 April 2004 - Accepted: 20 April 2004 - Published: 7 September 2004

\begin{abstract}
Induction magnetometer observations of dayside Pc1 activity at Barentsburg (BAB, Spitsbergen archipelago, $78.05^{\circ} \mathrm{N}, 14.12^{\circ} \mathrm{E}$ ) are combined with data from two magnetometers located in Scandinavia and the Kola peninsula. Seven events with very large negative IMF $B_{z}$ components were considered. For all of the events, the cusp location was expected to be significantly shifted equatorward from the statistical position such that the BAB magnetometer was located well inside the polar cap. The DMSP particle data indicated that the $\mathrm{BAB}$ magnetometer was indeed inside the polar cap, whereas other magnetometers were collocated with the ionospheric projections of the cusp, the low-latitude boundary layer or the boundary plasma sheet. Pc1 magnetic pulsations were observed only at BAB. In three cases, for which SuperDARN convection data were available, the Pc1 activity correlated with intervals of large-scale convection reconfiguration, such that the plasma flow crossing the $\mathrm{BAB}$ location was associated with newly-reconnected magnetic flux tubes drifting tailward. The convection reconfigurations were in response to a decrease in the IMF $B_{y}$ component. We argue that the source of the observed Pc1 pulsations is anisotropic plasma of the depletion layer within the magnetosheath. The plasma anisotropy supports the excitation of electromagnetic ion cyclotron waves that are detectable with a ground-based magnetometer when the flux tubes containing the unstable plasma become connected to the Earth's ionosphere in the course of the dayside reconnection processes.
\end{abstract}

Key words. Magnetospheric physics (MHD waves and instabilities; Plasma convection; Solar wind-magnetosphere interactions)

\section{Introduction}

Geomagnetic pulsations in the Pc1 frequency range of $0.1-$ $2 \mathrm{~Hz}$ have been observed on the ground at various latitudes.

Correspondence to: V. Safargaleev

(safar@pgi.kolasc.net.ru)
Their occurrence is often associated with the electromagnetic ion cyclotron (EMIC) waves driven by the temperature anisotropy of the magnetospheric plasma. Erlandson et al. (1990) and Anderson et al. (1992) showed that, in most cases, the source of pulsations is located in the inner magnetosphere where the EMIC instability can be initiated either by the injection of hot protons during magnetic storms (Kozyra et al., 1984; Erlandson et al., 1994; Engebretson et al., 2002) or by the squeezing of the magnetic flux tubes after compression of the magnetosphere (Olson and Lee, 1983; Engebretson et al., 2002). Of special interest are the high-latitude Pc1 pulsations where several types and sources of such waves have been identified. The discrete, short-lived Pc1 pulsations are mostly observed on the dayside, near the cusp area (Morris et al., 1985; Hansen et al., 1992; Neudegg et al., 1995; Sato and Fukunishi, 1999; Denton et al., 2002). For this reason, it was suggested that these Pc1 waves could be used to determine the location of the low-latitude boundary layer (llbl) or the cusp (Menk et al., 1992; Cole et al., 1982). The dayside, bursty ULF wave activity, similar in appearance to the nightside PiB pulsations, was related to poleward propagating multiple auroral forms (Kokubun et al., 1988). Pulsations with periodic modulation of the carrier frequency, the so-called "serpentine emissions", were observed in the polar cap, and they were related to certain structures in the solar wind (Dovbnya et al., 2002). Finally, a possibility was considered that dayside reconnection facilitates penetration of pulsations from the transition layer into the magnetosphere (Kato and Tonegawa, 1986; Lyatsky and Safargaleev, 1989). Clearly, studies of daytime high-latitude Pc1 magnetic pulsations can provide useful insights into the physics of the solar wind-magnetosphere interaction.

In space, Pc1 wave activity is often observed in that part of the transition layer adjacent to the dayside magnetopause and known as the plasma depletion layer or the magnetic barrier (Anderson and Fuselier, 1993; Denton et al., 1993). These pulsations have been attributed to the EMIC instability due to anisotropic plasma. The plasma depletion layer itself and the fact that the plasma is strongly anisotropic here 
are well known from satellite measurements (Crooker et al., 1979). One may regard the formation of this region as an inherent attribute of the solar wind-magnetosphere interaction (Zwan and Wolf, 1976; Rezhenov et al., 1995; Pudovkin et al., 2002).

In the course of dayside reconnection, the anisotropic plasma from the transition layer can appear on the magnetic field lines connected to the Earth's ionosphere in the nearcusp area such that magnetosheath-originated waves can be detected on the ground. When the IMF $B_{z}$ component is positive, reconnection between the interplanetary magnetic field (IMF) and geomagnetic field occurs poleward of the cusp, and the source of Pc1 pulsations happens to be inside the magnetosphere, on closed magnetic field lines (Lyatsky and Safargaleev, 1989). For this situation, an interpretation of the observations is not easy in the sense that one cannot say whether the pulsations result from reconnection or other processes. The high-latitude magnetic field lines converge towards lower altitudes, and therefore it is difficult to conclude from solely ground-based observations about the magnetospheric location of the Pc1 source. In addition, the waveguide properties of the ionosphere enable the transfer of Pc1 wave energy from its ionospheric entry points at middle latitudes to high latitudes (Morris et al., 1982). Conversely, when the IMF $B_{z}$ is negative, the source of Pc1 waves can be located on open field lines that are carried tailward by the solar wind after reconnection (Kato and Tonegawa, 1986). In this case, the association of the dayside ground-based Pc1 waves with reconnection is very likely.

In this paper we consider several Pc1 pulsations events in the dayside polar cap to show that their occurrence can be related to dayside reconnection. To achieve the goal, we analyze magnetic data from three significantly separated magnetic observatories together with IMF data from the WIND satellite and plasma convection obtained from SuperDARN radar measurements.

\section{Instrumentation and methods of investigation}

A key instrument for this study is the induction magnetometer operated at Barentsburg (BAB) on the Spitsbergen archipelago $\left(78.05^{\circ} \mathrm{N}, 14.12^{\circ} \mathrm{E}\right.$, geographic, $\mathrm{MLT}=\mathrm{UT}+2.4$ ). To investigate the possibility of pulsation propagation to $\mathrm{BAB}$ from lower latitudes through the ionospheric waveguide, data from magnetometers at Kilpisjärvi (KIL, $69.02^{\circ} \mathrm{N}, \quad 20.79^{\circ} \mathrm{E}$, geographic, $\mathrm{MLT}=\mathrm{UT}+2.0$ ) and Lovozero $\left(\mathrm{LOZ}, 67.97^{\circ} \mathrm{N}, 35.08^{\circ} \mathrm{E}\right.$, geographic, $\mathrm{MLT}=\mathrm{UT}+2.6)$ were analyzed. All magnetometers were operated at a sampling rate of $40 \mathrm{~Hz}$, so that studies of geomagnetic field variations in a frequency band from 0.1 to $20 \mathrm{~Hz}$ were possible. To visualize the temporal changes in the pulsation spectrum, FFT spectra averaged over $25 \mathrm{~s}$ with $\sim 5 \mathrm{~s}$ overlap of neighboring 25 -s intervals were employed.

Besides the possibility of electromagnetic wave propagation through the ionospheric waveguides, there is another factor complicating the interpretation of BAB Pc1 observa- tions. According to the statistics of Newell and Meng (1992), the cusp projection is typically located poleward of BAB. Thus, the BAB magnetometer would typically detect pulsations originating earthward of the magnetopause on closed magnetic field lines. Due to significant convergence of the magnetic field lines, the ionospheric projections of various magnetospheric regions are very narrow, and this makes it difficult to relate the source of pulsations to specific magnetospheric regions, although Morris et al. (1982) and Cole et al. (1982) reported cusp and llbl as the source regions for Pc1 pulsations. To identify the pulsation source location more correctly, Menk et al. (1992) and Hansen et al. (1992) performed an analysis of more extensive data sets that included satellite data. Also, Neudegg et al. (1995) were able to associate the Pc1 source with 1 lbl by applying the plane wavefront triangulation procedure.

For strongly negative IMF $B_{z}$, the cusp is shifted equatorward, so that $\mathrm{BAB}$ would be expected inside the polar cap, and therefore connected to open field lines, whereas KIL and LOZ would still stay on the closed field lines connected to llbl or the boundary plasma sheet (bps). Thus, the multi-station analysis allows one to study whether ionospheric waveguide propagation of pulsations takes place and to study the location of the Pc1 source with respect to specific magnetospheric regions. The absence of ULF activity at one of the observatories out several spatially separated ones enabled Kato and Tonegawa (1986) to relate Pc1s with the cusp and to estimate the signal attenuation as it propagates through the ionosphere. In our study, an absence of Pc1 pulsations at the low-latitude stations allowed us to associate the observed Pc1 waves at BAB with the open magnetic field lines convecting through the polar cap.

In this paper we consider events for which the IMF $B_{z}$ measured by the WIND satellite was large and negative. To define the BAB position relative to various magnetospheric regions, DMSP particle spectrograms were used. For three events, the ionospheric plasma convection was available from SuperDARN HF radar measurements. These latter measurements allowed us to discuss the observed Pc1s in the context of large-scale plasma circulation.

\section{Near-noon Pc1 bursts during the main phase of a magnetic storm on 28 October 2001}

\subsection{The background conditions}

On 28 October 2001 a magnetic cloud passed by the Earth and initiated a magnetic storm of about 150-nT intensity as measured by the $D_{s t}$ index (Fig. 1). Magnetic pulsations in the $\mathrm{Pc} 1$ range were observed during the main phase of this storm. Figure 1 shows the WIND data during the event. The time of the Pc1 activity at BAB is indicated by the vertical solid line. The leading front of the cloud reached the WIND location at $\sim 03: 12$ UT on 28 October, indicated by a sudden increase in the solar wind dynamic pressure of about $\sim 3 \mathrm{nPa}$. Eight minutes later, the SI-associated, step-like increase of 
the magnetic horizontal component was detected at the lowlatitude station Kakioka. This time delay is $\sim 5$ min smaller than the expected propagation time of the solar wind irregularity from the satellite to the magnetopause estimated from the formula given by Lester et al. (1993). We will use this formula for the estimation of propagation time for all events considered below keeping in mind that the error in the time lag may be as large as 5 minutes.

\subsection{Location of the magnetospheric domains}

During the event, two DMSP satellites flew above the stations almost simultaneously, allowing us to determine the location of the magnetometers relative to the boundary between the open and closed magnetic field lines. The distribution of precipitating particles was inferred from the spectrograms presented in Fig. 2. The boundaries of the inferred magnetospheric regions are indicated on the partial trajectories in Fig. 3a. It is seen that the cusp was shifted approximately by $10^{\circ}$ equatorward of its statistical position. The equatorward edge of the polar cap can be associated with the boundary between the cusp and mantle precipitations in the F12 data and with the boundary between the bps and polar rain precipitations in the F15 data. We can see that BAB was located on the open field lines, whereas the KIL position can be associated with the cusp or part of the llbl, east of the cusp. LOZ was equatorward of the llbl and bps ionospheric footprints. Note that there were no precipitations that might give auroras above the stations. This fact is important for interpretation of our observations, since the high-latitude Pc1 pulsations can be associated with the poleward moving auroral forms (Kokubun et al., 1988).

\subsection{Near-noon Pc1 bursts observed at BAB}

The spectrograms of ULF activity at all three stations are shown in Fig. 3b. Just after 08:30 UT Pc1 pulsations were observed at BAB (upper panel), located in the polar cap at this time, however, no pulsations were detected at KIL and LOZ, whose positions can be associated with the cusp/llbl and bps, respectively. This spatial distribution of Pc1 pulsations demonstrates that the pulsations at $\mathrm{BAB}$ were not a result of simple propagation through the ionospheric waveguide. Importantly, the Pc1 source was located on the open magnetic field lines.

The duration of the event was about $7 \mathrm{~min}$, the frequency of pulsations decreased slightly from $1.1 \mathrm{~Hz}$ at the beginning of interval to $0.6 \mathrm{~Hz}$ at the end. One can discern three consecutive bursts of activity.

Below we discuss several phenomena observed in space and on the ground in association with this Pc1 event to support our hypothesis that the observed Pc1 waves were related to the dayside reconnection. Later in Sect. 5 we consider a model that incorporates both spatial and spectral features of Pc1 pulsations.

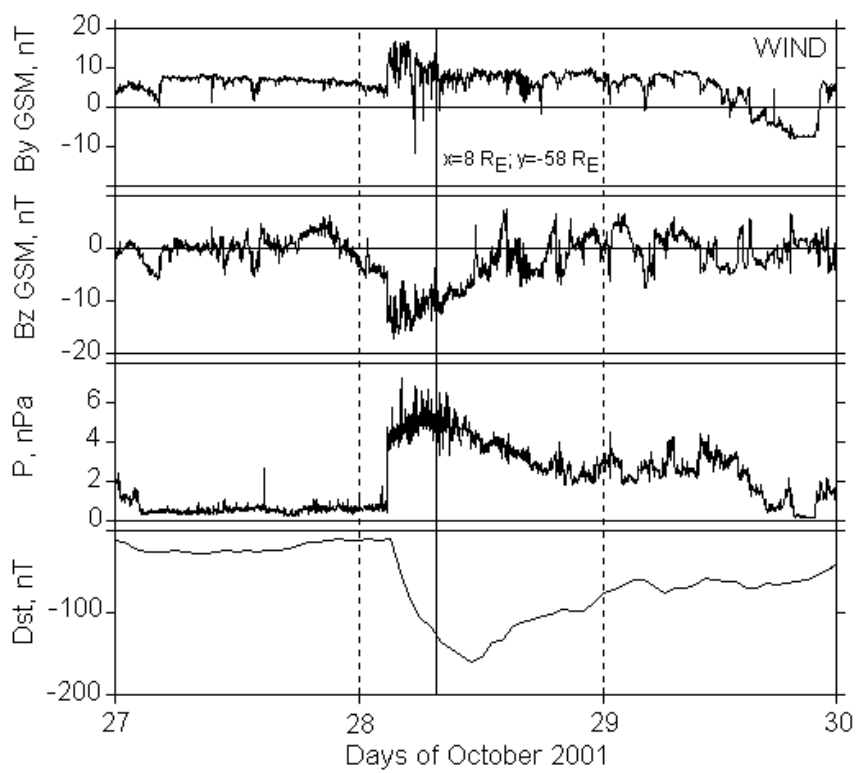

Fig. 1. IMF $B_{y}, B_{z}$ and dynamic pressure measured by the Wind satellite and $D_{s t}$ index for observations on 27 October-30 October 2001. The vertical line indicates the time of enhanced Pc1 activity in the polar cap.

\subsection{Changes in the IMF $B_{y}$ component prior to the event}

The three upper panels in Fig. 4 show the parameters of the interplanetary medium measured by the WIND satellite (measurements were shifted by the propagation time of 8 minutes). Prior to the Pc1 enhancement at BAB there was a decrease in the $B_{y}$ component, and the solar wind dynamic pressure experienced a series of impulses with amplitudes less than $2 \mathrm{nPa}$. Such pulses are very typical in the solar wind and they may not be considered as a trigger of reconnection. The decrease of $B_{y}$ to zero results in the reorientation of the $\mathrm{IMF}$ into the $\mathrm{XZ}$ plane. This indicates that just before the Pc1 event the merging point on the magnetopause shifted toward the noon meridian where the Earth's magnetic field has no significant y-component.

\subsection{Pc1 and Pc5 magnetic activities}

One of the phenomena accompanying the Pc1 event on the ground was a train of Pc5 magnetic pulsations observed by the IMAGE network. The pulsations had a regular form at all stations located on the Scandinavia peninsula but they were not seen poleward of the coast line. In Fig. 4 (lower panels) we present the magnetic field variations at two stations only, Kilpisjärvi (KIL) on the mainland and Longyearbyen (LYR) located $\sim 40$ kilometers east of BAB. The data from other IMAGE stations show that the amplitude of pulsations increases toward the northern coast of the peninsula with an absence of Pc5 pulsations at LYR. Rostoker et al. (1972) found that pulsations in the Pc5 range appeared to attain peak intensities at high latitudes and may be used to locate the cusp. Also, Arnoldy et al. (1996) connected such Pc5 pulsations 



Fig. 2. DMSP data for precipitating particles over Spitsbergen and Kola peninsula. Also indicated are the boundaries of the magnetospheric regions.

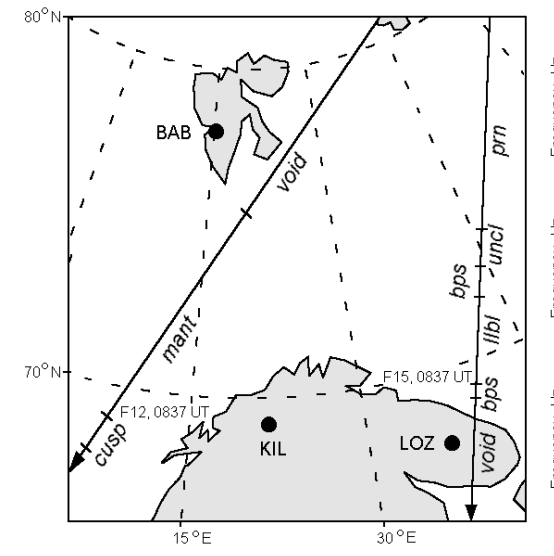

a

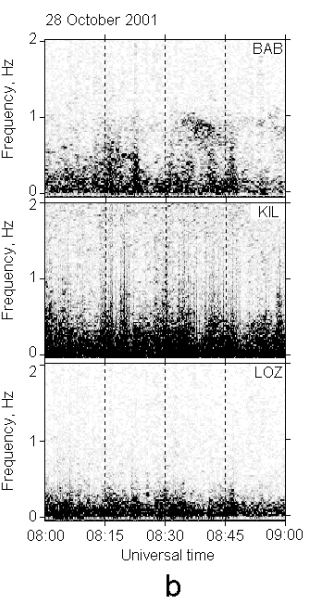

b

Fig. 3. (a) The location of the magnetic observatories BAB, KIL and LOZ are filled circles with the magnetospheric regions from DMSP F12 and F15 overlaid. (b) The spectrogram of ULF activity recorded at these observatories.

with a surface wave propagating along the magnetopause. Recently, Albes et al. (1998) proposed a method of distinguishing Alfvén and magneto-acoustic oscillations; this approach allows one to determine the boundary between the open and closed magnetic field lines more precisely. Because of the limited number of IMAGE magnetometers between $\mathrm{BAB}$ and the mainland, we cannot define the boundary location from IMAGE Pc5 data other than to say it is somewhat equatorward of LYR, which is an additional confirmation of our assumption that BAB is poleward of the cusp location.

3.6 Pc1 pulsations and enhancement of the Pykkvibaer radar line-of-sight velocity

Ionospheric conditons above $\mathrm{BAB}$ were monitored by two CUTLASS HF radars located at Hankasalmi (HANK) and Pykkvibaer (PYKK). These radars are sensitive to F-region plasma density irregularities with scale sizes of $10-15 \mathrm{~m}$. At F-region heights, these irregularities move with $\boldsymbol{E} \times \boldsymbol{B}$ drift such that the Doppler shift of the received signal is the lineof-sight (1-o-s) component of the convection velocity.

In Fig. 5 we present Pykkvibaer velocity data for three azimuthal scans around the interval of Pc1 activity at BAB. Before the event (upper panel of Fig. 5), the velocity was directed mostly away from the PYKK radar and had values of 300-500 m/s. At 08:35 UT (central panel of Fig. 5), two minutes prior to the Pc1 onset at BAB, the velocity had increased by a factor of 2-3. Pulsations stopped when the convection slowed down and echoes over BAB almost disappeared (bottom panel of Fig. 5). The Pc1 activity at BAB correlated with the interval of the enhanced Pykkvibaer 1-o-s velocity. The interval of enhanced convection is indicated in Fig. 4 by a horizontal bar.

3.7 Pc1 pulsations and reconfiguration of the large-scale convection pattern

A series of three diagrams in Fig. 6 shows the large-scale ionospheric convection patterns during the interval under investigation, including the period of the Pc1 event. The convection maps were obtained using data from all Northern Hemisphere SuperDARN radars with the map potential technique by Ruohoniemi and Baker (1998). Before the Pc1 enhancement (upper panel of Fig. 6), the convection was meridional west of BAB and more azimuthal, along the magnetic latitudes, close to it. The observatory (shown with the filled circle) was in an area of magnetic flux tubes drifting from 


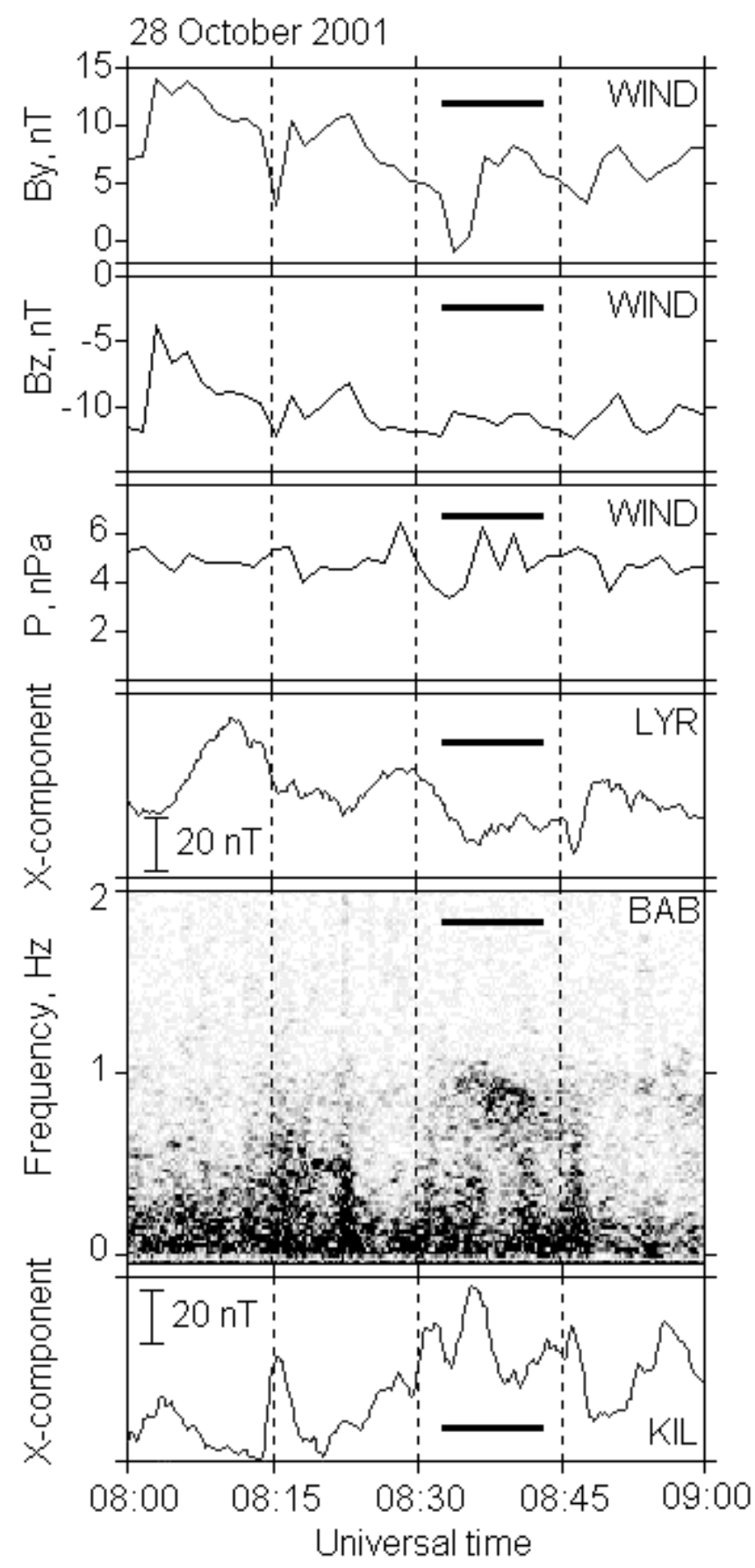

Fig. 4. The interplanetary magnetic field $\left(B_{z}, B_{y}\right)$, solar wind dynamic pressure $(\mathrm{P})$, magnetometer $\mathrm{X}$ component at IMAGE stations Kilpisjärvi (KIL) and Longyearbyen (LYR), and the Pc1 pulsations at Barentsburg (BAB) are presented.

the dusk sector. At the beginning of the Pc1 wave activity (middle panel), the character of convection changed dramatically. The convection was almost meridional and directed antisunward. In the middle panel, the dashed line shows the DMSP F12 footprint at the altitude of $300 \mathrm{~km}$. The open circle indicates the cusp position. The meridional plasma flow is an indication that newly-reconnected magnetic flux tubes
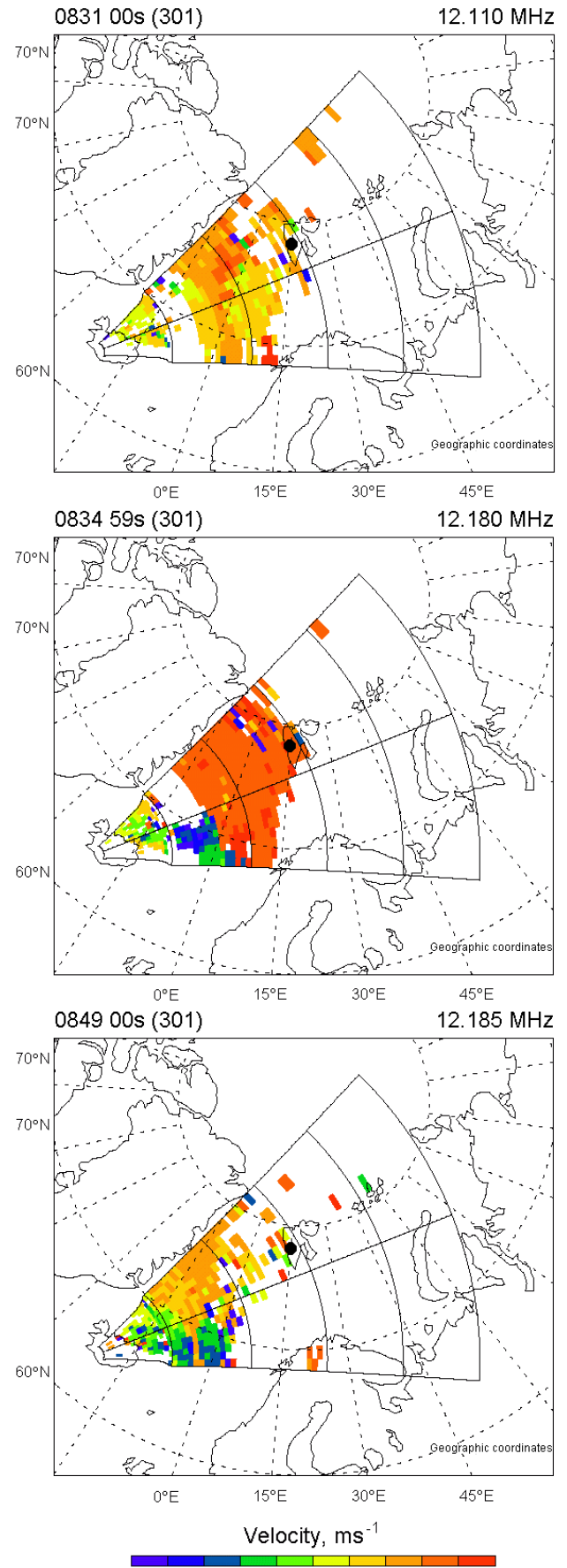

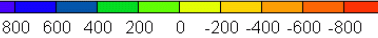

Fig. 5. Pykkvibaer HF radar data showing the line of sight velocity enhancement (negative velocities correspond to plasma motion away from the radar) during the $\mathrm{Pc} 1$ event at BAB.

drifting from the cusp area would have convected over BAB. From this point of view, the Pc1 activity in the polar cap can be related to penetration of the anisotropic plasma from the magnetosheath into the dayside magnetosphere. The lower panel in Fig. 6 corresponds to the time after the Pc1 event. There is evidence that the convection around $\mathrm{BAB}$ is once again azimuthal. 

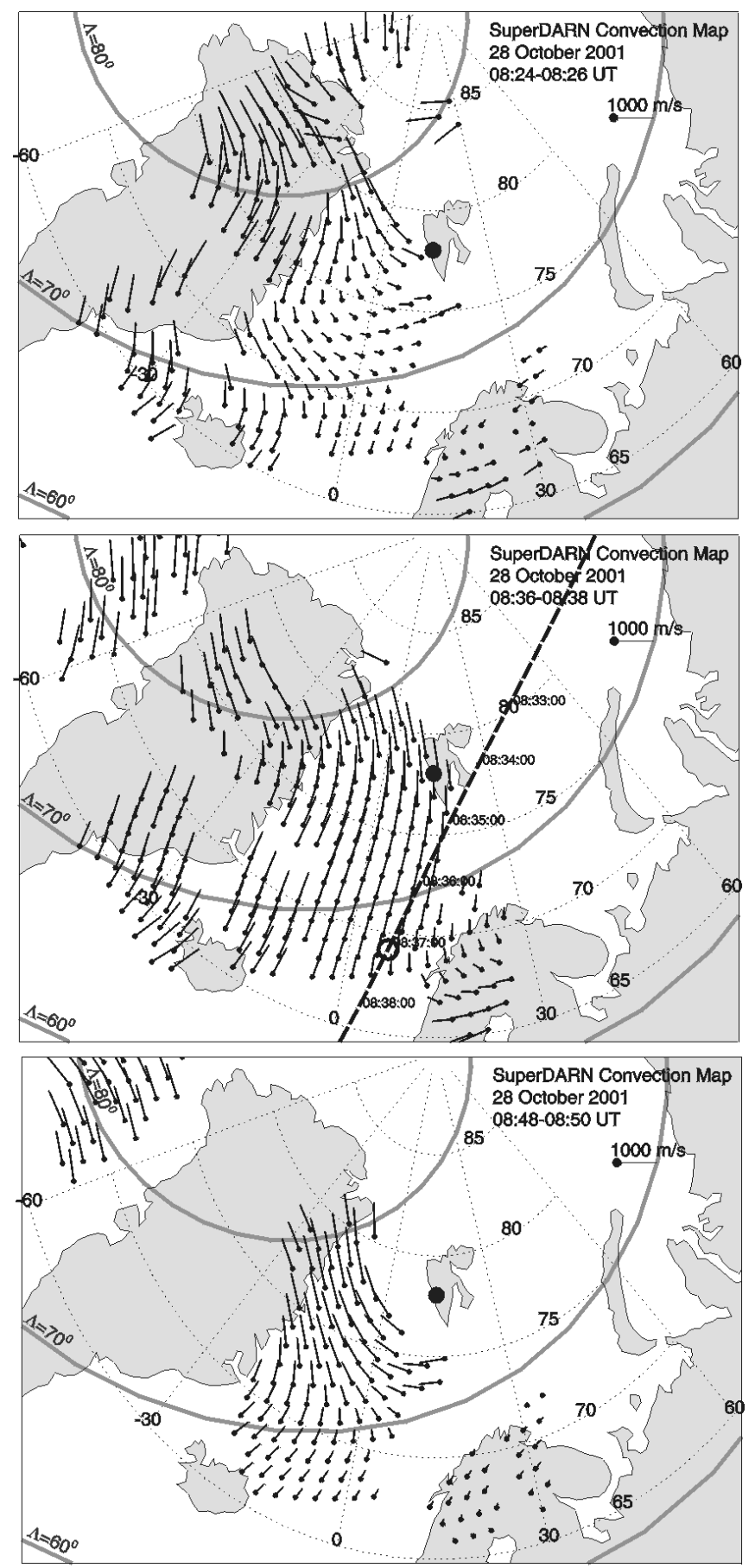

Fig. 6. Changes in the convection around $B A B$ as inferred from the SuperDARN measurements. The dashed line is a partial DMSP F12 trajectory. An open circle indicates the cusp position.

The convection rotation above $\mathrm{BAB}$ by almost $90^{\circ}$ during the Pc1 event were seen as an increase in the PYKK 1-o-s velocity, and both effects were the result of reconfiguration of the large-scale convection pattern. This is illustrated in Fig. 7, where the two-cell convection patterns inferred from SuperDARN measurements on a global scale are presented. Before the event, BAB was located under the dayside part
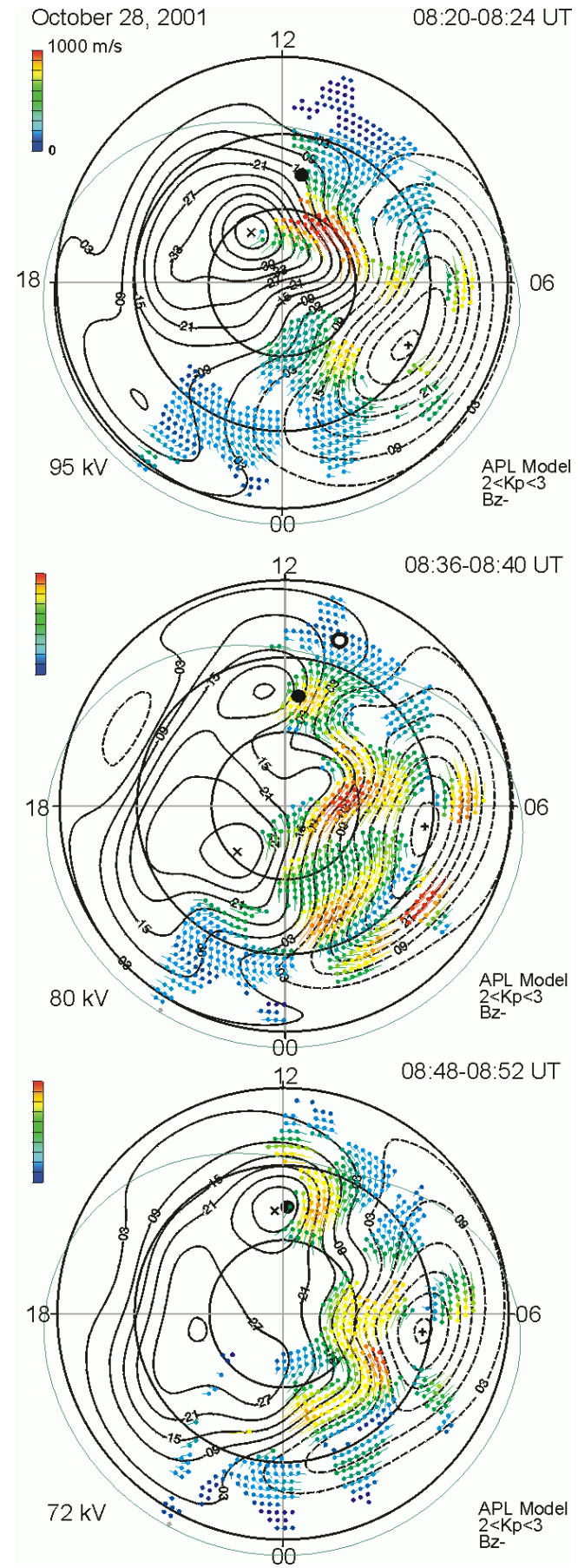

Fig. 7. The SuperDARN convection vectors showing the two-cell pattern of ionospheric convection before (top), during (middle) and after (bottom) the Pc1 event. The BAB location is indicated with filled circle. An open circle indicates the cusp position.

of the dusk cell. During the Pc1 event, the size of the dawn cell increased, the shape of the convection pattern became more symmetric, and $\mathrm{BAB}$ was within the convection flow separating the cells. After the event, the daytime dusk cell dominated again. Note that the above reconfiguration of the global-scale convection is consistent with expectations for a vanishing IMF $B_{y}$ component (Heelis, 1984). 



Fig. 8. Two examples of BAB Pc1 in which the frequency decreases with time. A plot illustrating the relationship of Pc1 event duration, $\Delta t$, and the IMF $B_{z}$ magnitude.

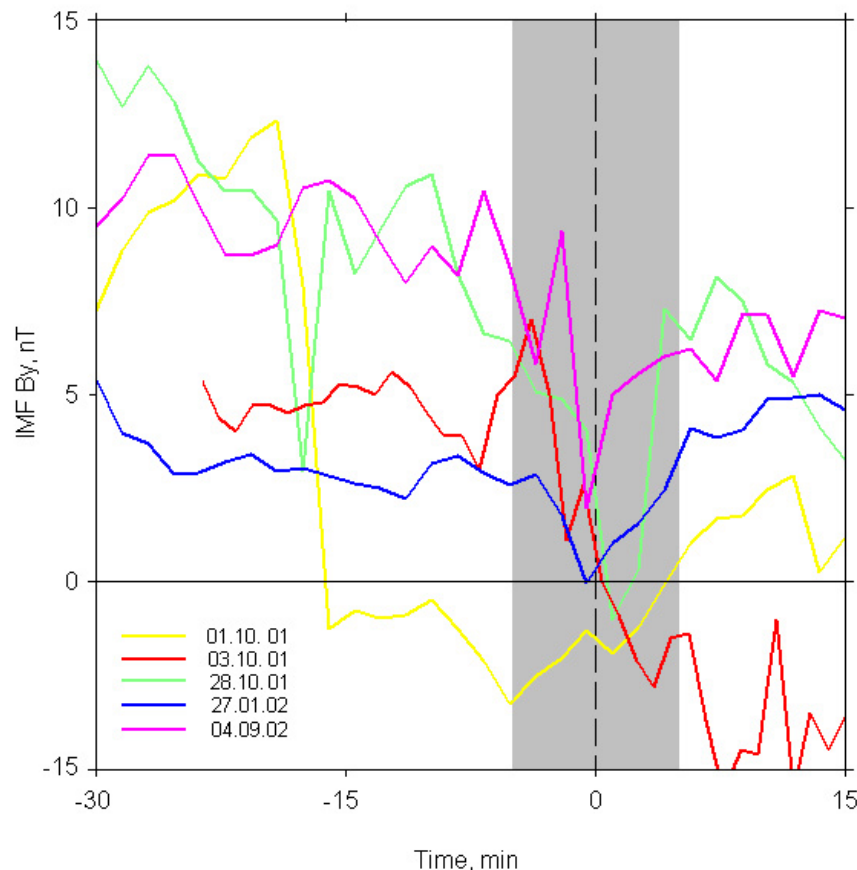

a



b

Fig. 9. (a) The results of superimposed epoch analysis for 5 events of the IMF $B_{y}$ component prior to the Pc1 enhancement. The shaded area is \pm 5 min from event onset. (b) The variation of the clock angle between the IMF $B_{y}$ and $B_{z}$ components for two Pc1 events.

\section{Pc1 pulsations for other events with large negative $B_{z}$}

To search for other events, intervals for which $\mathrm{BAB}$ was near local noon and where the IMF had large negative $B_{z}$ values were identified, for the period from October 2001 to October 2002. For such IMF conditions, the cusp footprint is expected to be at low latitudes, and therefore BAB is expected to be inside the polar cap. Concurrent DMSP data and Pc5 activity were used to support this assumption.
Table 1 lists the date, time, frequency and duration of the identified Pc1 pulsation events. In addition, the typical IMF $B_{z}$ values and solar wind conditions are presented, together with the peak $D_{s t}$ index (following the event), along with the availability of DMSP spectrograms. Also listed in Table 1 are two events for which no wave activity was observed. From the information presented in Table 1 one can make several conclusions. 


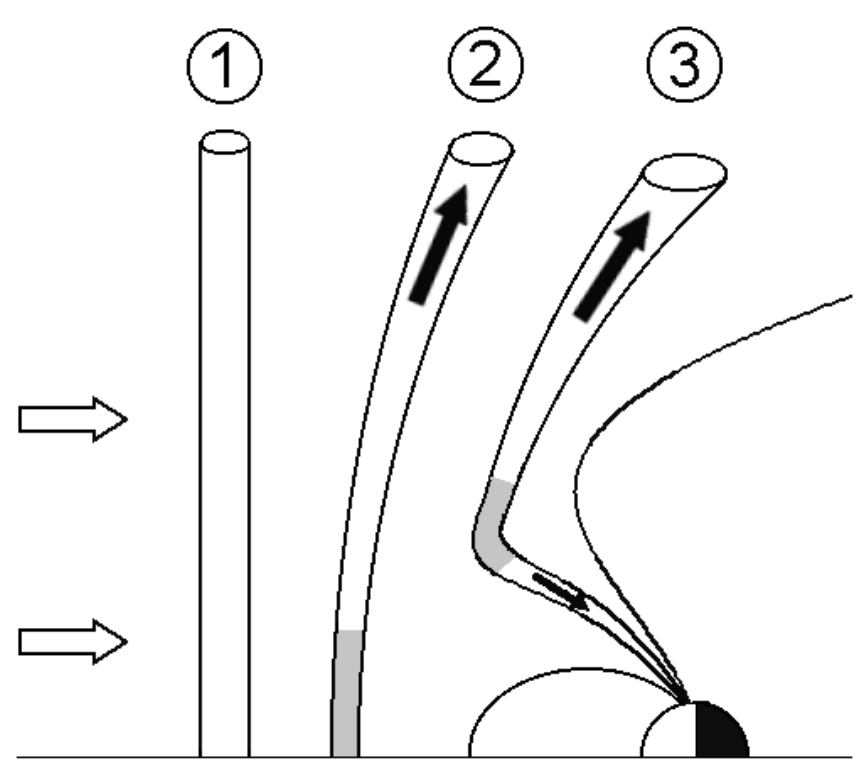

Fig. 10. Model of magnetosheath-originated EMIC waves (black arrows) penetration into the polar ionosphere. Tube 1 is the undisturbed magnetic flux tube in the solar wind. Tube 2 is the flux tube in the magnetic barrier (plasma depletion layer). Tube 3 is the reconnected flux tube. Anisotropic plasma cloud is shown in gray.

1. Pc1 pulsations were observed during the main phase of a magnetic storm caused by the passage of a magnetic cloud past the Earth.

2. For all selected events, the DMSP data showed that $\mathrm{BAB}$ was inside the polar cap, whereas the two other stations used in this study, KIL and LOZ, were on closed magnetic field lines. Their location can be associated with the ionospheric projections of llbl or bps.

3. In all cases, no Pc1 pulsations were detected at KIL and LOZ (near the $11 b l$ and bps projections).

4. The discrete ULF activity at BAB lasted for $3-18 \mathrm{~min}$ and had a form of single or several short-lived bursts. The event duration seems to be proportional to the magnitude of $B_{z}$ (see Fig. 8). The frequency of pulsations was slightly decreasing in the course of the events.

5. The decrease of the IMF $B_{y}$ component almost to zero took place within a 10-min interval near the time of pulsation enhancement. This is illustrated in Fig. 9a which shows the IMF $B_{y}$ variations at the subsolar point of the magnetopause. The time $\mathrm{T}=0$ corresponds to the beginning of $\mathrm{Pc} 1$ pulsations at $\mathrm{BAB}$. Highlighted is the interval of $0 \pm 5 \mathrm{~min}$, where $5 \mathrm{~min}$ is the uncertainty in determining the propagation time of the solar wind from the satellite to the subsolar point (see Sect. 3.1).

6. For the three events highlighted in Table 1 in gray, the SuperDARN data showed that near the time of Pc1s the orientation of convection flow near BAB became more meridional and antisunward than azimuthal. For the 28
October event (discussed above) such plasma motion very likely represented the newly-reconnected magnetic flux tubes drifting tailward from the cusp. For the two other cases (numbers 1 and 5 in Table 1) we were not able to determine the location of the cusp since the DMSP satellites were not in appropriate positions.

7. The train of Pc5 magnetic pulsations seen in the data of the IMAGE magnetometer network near the moment of Pc1 pulsations at BAB had the strongest amplitude at the station closest to the assumed location of the boundary between the open and closed magnetic field lines. No Pc5 pulsations were seen poleward of this boundary.

8. The DMSP data did not show electron precipitation which might cause discrete aurora above BAB.

9. For two periods with large negative $B_{z}$ reported at the bottom of Table 1 there was no Pc1 activity anywhere. Note that for these events, the IMF $B_{y}$ component did not decrease when BAB was located near local noon. Also, the solar wind dynamic pressure was relatively low, and this might be the reason why Pc1 waves were not generated in the magnetosheath.

\section{Discussion}

We have reported several cases of daytime Pc1 pulsation observations at Barentsburg (BAB) for which the observatory was located inside the polar cap. To make sure that measurements were indeed inside the polar cap, events with large negative IMF $B_{z}$ component were selected. This assumption was supported by the DMSP particle data and IMAGE magnetometer data allowing the proper determination of $\mathrm{BAB}$ and auroral zone observatories KIL and LOZ with respect to the boundary between the open and closed magnetic field lines. We excluded from consideration morning and evening hours because no satellite data were available and because we were not able to determine definitely the BAB location with respect to various magnetospheric regions by using IMAGE magnetometers (too few stations).

Before presenting a model explaining the onset of pulsations in the polar cap, let us review and discuss the observed features that the model should take into account. We showed that the pulsations were only detected in the polar cap. This observation rejects the possibility of ionospheric propagation of pulsations from the auroral zone latitudes to the polar cap, which implies that the source of pulsations should be located on the open magnetic field lines. Hence, even though the pulsations were observed under large negative $B_{z}$ (which resulted in the magnetic storm), it is unlikely that they originated from hot protons injected from the magnetotail plasma sheet, as assumed for some middle and high latitude Pc1s (Kozyra et al., 1984; Erlandson et al., 1994). Next, no sudden increases in the solar wind dynamic pressure and electron precipitation causing discrete aurora were associated with the pulsation events. Thus, the generation mechanism should be 
Table 1. Characteristics of intervals when BAB was inside the polar cap.

\begin{tabular}{|c|c|c|c|c|c|c|c|c|}
\hline No & $\begin{array}{l}\text { Date, } \\
\text { dd.mm.yy }\end{array}$ & UT & $\begin{array}{l}\mathrm{f}, \\
\mathrm{Hz}\end{array}$ & $\begin{array}{l}\mathrm{T}, \\
\min \end{array}$ & $\begin{array}{l}\mathrm{B}_{z} \\
\mathrm{nT}\end{array}$ & $\begin{array}{l}\mathrm{P}, \\
\mathrm{nPa}\end{array}$ & $\left|D_{s t}\right|, \mathrm{nT}$ & $\begin{array}{l}\text { DMSP, } \\
\text { UT }\end{array}$ \\
\hline 1 & 2 & 3 & 4 & 5 & 6 & 7 & 8 & 9 \\
\hline 1 & 01.10 .01 & 0850 & 0.7 & 10 & -10 & 3 & 150 & $\mathrm{~F} 14,0848$ \\
\hline 2 & 03.10 .01 & 0914 & 0.9 & 18 & -21 & - & 180 & $\mathrm{~F} 15,0938$ \\
\hline 3 & 28.10 .01 & 0833 & 0.9 & 7 & -11 & 5 & 160 & $\mathrm{~F} 13,0837$ \\
\hline 4 & 27.05 .02 & 0845 & 1.3 & 3 & -7 & 2 & 60 & $\mathrm{~F} 14,0816$ \\
\hline 5 & 04.09 .02 & 0915 & 0.5 & 12 & -12 & 3 & 100 & $\mathrm{~F} 15,0919$ \\
\hline 6 & 02.02 .02 & - & - & - & -10 & 1.5 & 80 & $\mathrm{~F} 14,0917$ \\
\hline 7 & 18.04 .02 & - & - & - & -11 & 0.6 & 125 & $\mathrm{~F} 14,0711$ \\
\hline
\end{tabular}

different from those discussed, for instance, by Fukunushi et al. (1981) and Kokubun et al. (1988). The dynamic spectrum of Pc1s at BAB were similar to "irregular pulsations of raising period" (IPRP) observed by Morris et al. (1982) near the llbl projection. These authors suggested that the IPRP might be generated by hot protons which were captured along the magnetopause and had become unstable after mixing with the cold plasma of the ionospheric origin (Cole et al., 1982). Since the source of IPDP was assumed to be on closed magnetic field lines, the above mechanism cannot be applied to our cases. The so-called "serpentine emissions" (SE) were found definitively in the area of open magnetic field lines (Dovbnya et al., 2002), but the dynamic spectrum of SE differs significantly from that of pulsations observed at BAB.

It is widely accepted that the anisotropic proton plasma can be a source of Pc1 pulsations. As the magnetospheric lobes lack any plasma, the question is where do the anisotropic protons come from? We think that dayside reconnection might bring the protons to the lobes. The absence of Pc1 pulsations at KIL and LOZ indicates that no ionospheric propagation occurred from high to lower latitudes. Similar observations were reported previously by Neudegg et al. (1995) and Sato and Fukunishi (1999). These authors did not observe discrete Pc1 bursts at several observatories located close to the one at which the pulsations were seen. They suggested that attenuation of the waves in the ionospheric waveguide was too high (of the order of $10 \mathrm{~dB} / 100 \mathrm{~km}$, in accordance with estimations by Neudegg et al., 1995) to detect signals at close locations. Taking the large damping into account, Sato and Fukunishi (1999) concluded that the observed Pc1 pulsations can only be excited within a limited area. So the absence of these waves equatorward of BAB suggests that we also have locally originated pulsations. By assuming that the source of Pc1 pulsations moves together with the convection, the data set for the 28 October event allows us to estimate the spatial extension of an area on the ground where pulsations can still be detected. As was mentioned in Sect. 3.3, the Pc1 activity has a form of several bursts of 2-3 min duration (similar behavior is demon- strated for the events of Fig. 8). During the event, the convection velocity above $\mathrm{BAB}$ was about $1 \mathrm{~km} / \mathrm{s}$. Combination of these two values gives the size of an area of $120-180 \mathrm{~km}$. This is smaller than the distance between KIL and the projection of the cusp in the ionosphere, which was $\sim 500 \mathrm{~km}$. Neudegg et al. (1995) obtained an estimates of $\sim 300 \mathrm{~km}$ for the distance over which pulsations would disappear completely. On the other hand, this is of the same order as the size of the ionospheric projection of FTEs observed by Rijnbeek et al. (1984) in the vicinity of the magnetopause which may be considered as the size of the reconnected flux tubes.

We cannot explain the absence of discrete Pc1 waves at KIL and LOZ by poor ionospheric propagation conditions, since the broad-band magnetic noise below $0.5 \mathrm{~Hz}$ behaved similarly at BAB, KIL and LOZ, namely, the 15-min recurrence of signal bursts, as seen in Fig. 3b. This similarity indicates that this noise activity originated from one place in the magnetosphere and then propagated in the ionospheric waveguide. The series of ULF enhancements at KIL correlates well with an increase in the $\mathrm{H}$-component at this station (Fig. 4, bottom panel) which is in agreement with Sato and Fukunishi (1999), who associated such activity with magnetic impulse events and mentioned that in contrast to discrete $\mathrm{Pc} 1 \mathrm{~s}$, the broad-band pulsations can propagate to large distances.

One more significant feature of this paper is the special IMF conditions corresponding to the occurrence of Pc1 waves. This distinguishes our observations from those reported by Cole et al. (1982), who found no correlation between IPRP events and the state of IMF. The most important result is the correlation of $\mathrm{Pc1}$ pulsations with a decrease in the IMF $B_{y}$ component (Fig. 9a). Pulsations were observed near local noon and when the IMF magnetic field lines were mostly in the XZ plane. This suggests a possible relation of midday Pc1 pulsations with the reconnection process. The SuperDARN data support this hypothesis; they show that, during the Pc1 events the BAB magnetometer was located under flux tubes drifting across the polar cap from the dayside (very likely from the cusp) to the night-side. Also, we 
should mention the correlation of the event duration with the IMF $B_{z}$ magnitude (see Fig. 8, right panel). Finally, we note that the model should also explain the frequency-time dependence of the observed Pc1 spectrum.

We propose the following model for the excitation of Pc1 pulsations that encompasses all of the above listed features (see sketch in Fig. 10). We assume that the EMIC waves (which are the Pc1 pulsations) are first excited in the plasma depletion layer due to the presence of anisotropic plasma. However, these waves cannot be detected by the groundbased magnetometers as they are located outside the magnetosphere. The reduction of the IMF $B_{y}$ component to zero makes the IMF field lines parallel to the geomagnetic field lines that form the magnetopause near the noon meridian. This creates favorable conditions for the flux lines with the unstable plasma to be connected to the Earth's ionosphere via reconnection, and as a consequence the magnetosheath originated EMIC waves are now detectable at the ground in the form of Pc1 bursts. Moreover, the decrease in the IMF $B_{y}$ component would result in improved conditions for $\mathrm{BAB}$ observations on those flux tubes that drift from the cusp towards the tail, since for small IMF $B_{y}$ the ionospheric convection cells become more symmetric (Heelis, 1984) and the flux tubes can convect over the observatory location.

The correlation of the Pc1 event duration with IMF $B_{z}$ can be understood within the above model if one assumes that the duration depends on the time that BAB stays under the reconnected tailward-drifting flux tubes. The duration depends on the amount of time that the IMF vector has the necessary orientation, i.e. almost in XZ plane. Larger $B_{z}$ values would yield longer intervals for which the angle between $B_{z}$ and varying $B_{y}$ would be small enough to consider that the full vector is almost in the XZ plane. In general, our data demonstrate such a tendency. Figure $9 b$ presents the angle between $B_{y}$ and $B_{z}$ as a function of time for two events which occurred under the largest (red line) and the smallest (blue line) values of IMF $B_{z}$. The time T=0 corresponds to the beginning of $\mathrm{Pc} 1$ pulsations at $\mathrm{BAB}$. An angle of zero degrees represents an IMF vector in the XZ plane. For the "long" Pc1 event which occurred at large $B_{z}$, the IMF vector was close (within $\pm 10^{\circ}$ ) to the XZ-plane for a relatively long period as compared with the "short" duration event observed at smaller $B_{z}$.

We can also explain the fine structure of Pc1 pulsations and why the frequency of pulsations decreased with time if we assume that reconnection occurs in an extended area such that various reconnected flux tubes take different times to reach the $\mathrm{BAB}$ magnetometer. Within a specific newlyreconnected flux tube, the anisotropic plasma will be pushed away from the equatorial plane toward the area in the magnetosheath where the flux tubes are less compressed and the magnetic field is smaller. This would result in a decrease in the local proton cyclotron frequency (as well as the frequency of EMIC waves). This implies that a flux tube arriving at $\mathrm{BAB}$ later would produce a new burst of Pc1s at a lower frequency, exactly what is observed.
In the preceding explanation, one may wonder about the reason for the plasma being squeezed away from the equatorial plane. This might occur because of the transformation of the magnetic energy into plasma kinetic energy in the course of reconnection. Under typical magnetosheath conditions $\left(\mathrm{p}_{\perp}>\mathrm{p}_{\|}\right)$, the plasma can be accelerated along the magnetic field lines to a maximum speed of $1.5 \mathrm{~V}_{A}$ (Mühlabacher et al., 2003). Here $\mathrm{p}_{\perp}$ and $\mathrm{p}_{\|}$are the proton pressures across and along the magnetic field, respectively, and $\mathrm{V}_{A}$ is the Alfvén speed. Using the AMPTE/IRM satellite data summarized by Phan et al. (1994), one can estimate the Alfvén velocity near the magnetopause to be $\sim 200 \mathrm{~km} / \mathrm{s}$. The simple model of the magnetosheath used by Rezenov et al. (1995) for calculation of the anisotropy ratio also allows us to estimate the gradient of the magnetic field along a magnetic flux tube. For typical values of the solar wind velocity of $\sim 400 \mathrm{~km} / \mathrm{s}$, the distance between the bow shock and the magnetopause of $\sim 6 \mathrm{R}_{E}$ and the propagating time from the bow shock to the magnetopause of $\sim 7 \mathrm{~min}$., one obtains the decrease of $\mathrm{B}$ by a factor of 3 over a distance of $\sim 30 \mathrm{R}_{E}$. Assuming the frequency of $\mathrm{Pc} 1$ to be proportional to $\mathrm{B}$, one obtains a factor of 1.5 in reduction of the frequency per 5 minutes. This estimate agrees well with the data presented in Fig. 8.

Finally, we would like to mention dayside reconnection as a possible reason for the cusp-associated Pc1 pulsations as previously discussed by Kato and Tonegawa (1986). We applied their model to observations in the polar cap and made simple estimates to support it based upon our observational conditions. However, there are at least two difficulties with this model that we would like to mention here. First of all, it is important to estimate quantitatively the effect of reconnection on the development of anisotropy. Mühlabacher et al. (2003) demonstrated recently how the pressure anisotropy influences dayside reconnection, but nothing has been said on the effect of reconnection on the anisotropy of the plasma. Insights into this effect would be crucial for our model. Second, the presence of a plasma depletion layer is important for the creation of anisotropic plasma. Presently, however, there is no common opinion on the possibility of its formation during negative IMF $B_{z}$ (Pudovkin et al., 2002). In this view, it is worth mentioning an alternative model of Pc1 generation which is not based on the proton anisotropy in the magnetosphere. The so-called "ionospheric Alfvén resonator" (IAR) could be the reason for some types of ULF activity in the Pc1 range (Demekhov et al., 2000). The difficulty with this model is that the properties of IAR in the polar ionosphere are not well investigated and also there were reports on the non-ionospheric nature of near-cusp Pc1s, e.g. Hansen et al. (1992).

\section{Conclusion}

Using data from three properly spaced high-latitude magnetic stations we examined the dayside Pc1 magnetic pulsations for cases in which the most poleward station (Barentsburg) was located in the polar cap. The events appeared 
to correlate with a decrease in the IMF $B_{y}$ component to almost zero and the resulting reconfiguration of the large-scale ionospheric convection. It turned out that for the events considered here the Barentsburg magnetometer was able to detect signals associated with the magnetic flux tubes drifting tailward from the noon sector, and probably from the cusp. To explain the observations we assumed that the source of the observed Pc1 pulsation was the EMIC instability of the anisotropic plasma from the magnetosheath penetrating into the dayside magnetosphere as a result of reconnection.

Acknowledgements. We thank A. Voronin and S. Noskov (PGI) for $\mathrm{BAB}$ and LOZ data of good quality, and N. Kudryashova (PGI) for assistance in the data processing. V.S. is grateful to M.Yamauchi, H. Nilson and T.Sergienko (IRF, Sweden) for useful discussion. The data of the KIL induction magnetometer were provided by SGO (Finland). The solar wind and IMF data from the WIND satellite were obtained from the CDAWeb ISTP Key Parameter database (data providers are K. Olgivie and R. Lepping of NASA). CUTLASS is a PPARC facility deployed and operated by the University of Leicester in cooperation with the Finish Meteorlogical Institute, Helsinki and the Institute for Space Physics, Uppsala. The northern hemisphere SuperDARN radars are funded through the research agencies of Canada, France the UK and the USA. We thank the PIs of the radars used here for the use of the data. The LYR and KIL magnetic stations are a part of the IMAGE system. The DMSP particle detectors were designed by D. Hardy of AFRL, and the particle data were obtained from JHU/APL. We thank Dave Hardy, Fred Rich, and Patrick Newell for making them available. The KAK magnetic data and $D_{s t}$ index are from the Kyoto World Data Center C-2 in Kyoto, Japan. The work of V.S. was partly supported by Svenska Institutet (VISBI Programm stipend). A.V.K. acknowledges funding from NSERC (Canada).

Topical Editor T. Pulkkinen thanks B. Fraser and another referee for their help in evaluating this paper.

\section{References}

Ables, S. I., Fraser, B. J., Waters, C. L., and Neudegg, D. A.: Monitoring cusp/cleft topology using Pc5 ULF waves, Geophys. Res. Lett., 25, 1507-1510, 1998.

Anderson, B. J., Erlandson, R. E., and Zanetti, L. J.: A statistical study of Pc 1-2 magnetic pulsations in the equatorial magnetosphere: 1. Equatorial occurrence distribution, J. Geophys. Res., 97, 3075-3088, 1992.

Anderson, B. J., and Fuselier, S. A.: Magnetic pulsations from 0.1$4.0 \mathrm{~Hz}$ and associated plasma properties in the Earth's subsolar magnetosheath and plasma depletion layer, J. Geophys. Res., 98, 1461-1479, 1993.

Arnoldy, R. L., Engebretson, M. J., and Cahill, L. J., Jr.: Bursts of Pc 1-2 near the ionospheric footprint of the cusp and their relationship to flux transfer events, J. Geophys. Res., 93, 10071016, 1988.

Arnoldy, R. L., Engebretson, M. J., Alford, J. L., Erlandson, R. E., and Anderson, B. J.: Magnetic impulse events and associated Pc1 bursts at dayside high latitude, J. Geophys. Res., 101, 77937799, 1996.

Cole, K. D., Morris, R. J., Matveeva, E. T., Troitskaya, V. A., and Pokhotelov, O. A.: The relationship of the boundary layer of the magnetosphere to IPRP events, Planet. Space Sci., 30, 129-136, 1982.
Crooker, N. U., Eastman, T. E., and Stiles, G. S.: Observation of plasma depletion layer in the magnetosheath at the dayside magnetopause, J. Geophys. Res., 84, 869-874, 1979.

Demekhov, A. G., Trakhtengerts, V. Yu., and Bösinger, T.: Pc 1 waves and ionospheric Alfvén resonator: generation or filtration? Geophys. Res. Lett., 27, 3805-3808, 2000.

Denton, R. E., Hudson, M. K., Fuselier, S. A., and Anderson, B. J.: Electromagnetic ion cyclotron waves in the plasma depletion layer, J. Geophys. Res., 98, 13 477-13 490, 1993.

Denton, R. E., LaBelle, J., and Zhu, X.: Location of Pc1-2 waves relatively to the magnetopause, Ann. Geophys., 20, 1763-1767, 2002.

Dovbnya, B. V., Matveeva, E. T., Parkhomov, V. A., Ruban, V. F., and Shchepetnov R. V.: Diagnostics of flare-related solar wind streams based on high-latitude observations of hydromagnetic emissions of varying frequency, Geomagnetism and Aeronomy, 42, 1, 88-93, 2002.

Engebretson, M. J., Peterson, W. K., Posch, J. L., Klatt, M. R., Anderson, B. J., Russel, C. T., Singer, H. J., Arnoldy, R. L., and Fukunishi, H.: Observation of two types of Pc1-2 pulsations in the outer dayside magnetosphere, J. Geophys. Res., 107, SMP20, 2002.

Erlandson, R. E., Zanetti, L. ., Potemra, T. A., Block, L. R., and Holmgren, G.: Viking magnetic and electric observations of Pc 1 waves at high latitudes, J. Geophys. Res., 95, 5941-5955, 1990.

Erlandson, R. E., Zanetti, L. J., Engebretson, M. J., Arnoldy, R. L., Bösinger, T., and Mursula, K.: Pc1 waves generated by a magnetospheric compression during recovery phase of a geomagnetic storm, in Solar Wind Sources of Magnetospheric UltraLow-Frequency Waves, (Eds) Engebretson, M. J., Takahashi, K., and Scholer, M., AGU, Washington D.C., 1994.

Fukunishi, H., Toya, T., Koike, K., Kuwashima, M., and Kawamura, M.: Classification of hydromagnetic emissions based on frequency-time spectra, J. Geophys. Res., 86, 9029-9039, 1981.

Hansen, H. J., Fraser, B. J., Menk, F. W., Hu, Y.-D., Newell, P. T., Meng, C.-I., and Morris, R. J.: High-latitude Pc1 bursts arising in the dayside boundary layer region, J. Geophys. Res., 97, 39934008, 1992.

Heelis, R. A.: The effect of interplanetary magnetic field orientation on dayside high-latitude ionospheric convection, J. Geophys. Res., 89, 2873-2880, 1984.

Kangas, J., Guglielmi, A., and Pokhotelov, O.: Morphology and physics of short-period magnetic pulsations, Space Sci. Review, 83, 435-512, 1998.

Kato, Y. and Tonegawa, Y.: Pc1 pulsations observed at Cambridge Bay in the cusp region and Fort Smith in the auroral region, Proceedings of the eighth symposium on coordinated observation of the ionosphere and magnetosphere in the polar regions, in Memoirs of National Institute of Polar Research, Special Issue, No 42, p. 52, (Ed) Sato, N., Japan, 1986.

Kokubun, S., Yamamoto, T., Hayashi, K., Oguti, T., and Egeland, A.: Impulsive Pi bursts associated with poleward moving auroras near the polar cusp, J. Geomag. Geoelectr., 40, 537-551, 1988.

Kozyra, J. U., Cravens, T. E., Nagy, A. F., Fontheim, E. G., and Ong, R. S.: Effects of energetic heavy ions on the electromagnetic ion cyclotron wave generation in the plasmapause region, J. Geophys. Res., 89, 2217-2234, 1984.

Lester, M., De la Beaujardière, O., Foster, J. C., Freeman, M. P., Lühr, H., Ruohoniemi, J. B., and Swider, W.: The response of the large scale ionospheric convection pattern to changes in the IMF and substorms: results from the SUNDIAL 1987 campaign, Ann. Geophys., 11, 556-571, 1993. 
Lyatsky, W. B. and Safargaleev, V. V.: Generation of pulsation in Pc1 range in the cusp area, Geomagnetism and Aeronomy, 29, 512-513, 1989 (Engl. Transl.).

Menk, F. W., Fraser, B. J., Hansen, H. J., Newell, P. T., Meng, C.-I., and Morris, R. J.: Identification of magnetospheric cusp and cleft using Pc1-2 ULF pulsations, J. Atmos. Terr. Physics, 54, No 7-8, 1021-1042, 1992.

Morris, R. J., and Cole, K. D.: Pc 1-2 discrete regular daytime pulsation bursts at high latitudes, Planet. Space Sci., 33, 53-67, 1985.

Morris, R. J., Cole, K. D., Matveeva, E. T., and Troitskaya, V. A.: Hydromagnetic "wistles" at the dasyde cusps - IPRP events, Planet. Space Sci., 30, 113-127, 1982.

Mühlabacher, S., Ivanova, V.V., Semenov, V.S., Biernat, H.K., Langmayer, and D., Vogl, D.F.: Time depended reconnection for anisotropic pressure, Physics of Plasma, 10, 655-663, 2003.

Newell, P. T. and Meng, C.-I.: Mapping the dayside ionosphere to the magnetosphere according to particle precipitation characteristics, Geophys. Res. Lett., 19, 609-612, 1992.

Neudegg, D. A., Fraser, B. G., Menk, F. W., Hansen, H. J., Burns, G. B., Morris, R. J., and Underwood, M. J.: Sources and velocities of Pc1-2 ULF waves at high latitudes, Geophys. Res. Lett., 22, 2965-2968, 1995.

Olson, J. V. and Lee, L. C.: Pc1 wave generation by sudden impulses, Planet. Space Sci., 31, 295-302, 1983.
Phan, T.-D., Paschman, G., Baumjohann, W., Scopke, N., and Lühr, H.: The magnetosheath region adjacent to the dayside magnetopause: AMPTE/IRM observations, J. Geophys. Res., 99, 121141, 1994.

Pudovkin, M. I., Zaitseva, S. A., Lebedeva, V. V., Samsonov, V. A., Besser, B. P., Meister, C.-V., and Baumjohann, W.: MHDmodeling of the magnetosheath, Planet. Space Sci., 50, 473-488, 2002.

Rezhenov, B. V., Safargaleev, V. V., and Lyatsky, W. B.: On the formation of a plasma pressure anisotropy in the dayside magnetosheath, Ann. Geophys., 13, 237-241, 1995.

Rijnbeek, R. P., Cowley, S. W. H., Southwood, D. J., and Russel, C. T.: A survey of dayside flux transfer events observed by ISEE 1 and 2 magnetometers, J. Geophys. Res., 89, 786-800, 1984.

Rostoker, G., Samson, J., and Higuchi, Y.: Occurrence of Pc4,5 micropulsation activity at the polar cusp, J. Geophys. Res., 77, 4700-4706, 1972.

Ruohoniemi, J. M. and Baker, B.: Large-scale imaging of high latitude convection with Super Dual Auroral Radar Network HF radar observations, J. Geophys. Res., 103, 20 797-20 812, 1998.

Sato, M. and Fukunishi, H.: Magnetic impulse event and related Pc1 bursts observed by the automatic geophysical observatories network in Antarctica, J. Geophys., Res., 104, 19971-19982, 1999.

Zwan, B. J. and Wolf, R. A.: Depletion of solar wind plasma near a planetary boundary, J. Geophys. Res., 81, 1636-1648, 1976. 